\title{
Finite thermal diffusivity at onset of convection in autocatalytic systems: Discontinuous fluid density
}

\author{
Desiderio A. Vasquez \\ Department of Physics, Indiana University-Purdue University Fort Wayne, \\ Fort Wayne, Indiana 46805-1499 \\ Boyd F. Edwards \\ Department of Physics, West Virginia University, P.O. Box 6315, Morgantown, West Virginia 26506-6315 \\ Joseph W. Wilder \\ Department of Mathematics, West Virginia University, P.O. Box 6310, Morgantown, \\ West Virginia 26506-6310
}

(Received 22 October 1993; accepted 21 June 1995)

A linear convective stability analysis for propagating autocatalytic reaction fronts includes density differences due to both thermal and chemical gradients. Critical parameters for the onset of convection are calculated for an unbounded geometry, a vertical slab, and a vertical cylinder. Thermal effects are important at unstable wavelengths well above the critical wavelength for the onset of convection. (C) 1995 American Institute of Physics.

Experiments observe convection for ascending iodatearsenous acid fronts in vertical tubes whose diameters exceed a critical diameter of about $1 \mathrm{~mm} .{ }^{1,2}$ Linear hydrodynamic stability theory ${ }^{3,4}$ predicts a transition to buoyancy-driven convection with a critical diameter that agrees with these experiments, although large experimental and theoretical uncertainties preclude a precise comparison. A $24 \%$ theoretical uncertainty is due to the difference between the critical diameters calculated for infinite and zero thermal diffusivity. To reduce this uncertainty, calculations are needed at finite thermal diffusivity.

The equation of state $\rho(T)=\rho_{1} \gamma\left[1-\alpha\left(T-T_{1}\right)\right]$ is a first-order Taylor expansion of the temperature-dependent mass density about $T_{1}$, the final adiabatic temperature of the reacted fluid. Here, $\gamma=1$ in the unreacted fluid and $\gamma=1-\delta$, in the reacted fluid to account for chemically driven density changes, and $\alpha$ is the isobaric thermal expansion coefficient.

Neglecting small density changes $\delta_{1} \approx \alpha \Delta T \approx 1 \times 10^{-4}$ except where they modify gravity to first order allows us to write the dimensionless thermohydrodynamic equations of motion as

$$
\begin{aligned}
& \frac{\partial \mathbf{v}}{\partial t}+(\mathbf{v} \cdot \nabla) \mathbf{v}=-\mathscr{H}_{C}\left(T_{1}-T\right) \hat{z}-\nabla P+\nabla^{2} \mathbf{v}, \\
& \nabla \cdot \mathbf{v}=0,
\end{aligned}
$$

and

$$
\frac{\partial T}{\partial t}+v \cdot \nabla T=g_{T} \nabla^{2} T
$$

These are measured in units of length $a$ to be specified below, time $a^{2} / \nu$, pressure $\rho_{1} \nu^{2} / a^{2}$, and temperature $\delta_{1} / \alpha$, where $\nu$ is the kinematic viscosity. The convenient reduced pressure $P$ is related to the conventional pressure by $P=P_{\text {conv }}+\left(\gamma g a^{3} / \nu^{2}\right) z$. The equations also involve a dimensionless thermal diffusivity $\mathscr{D}_{T}=D_{T} / \nu$, a dimensionless molecular diffusivity $\mathscr{L}_{C}=D_{C} / \nu$, and a dimensionless driving parameter $\mathscr{F}=\delta_{1} a^{3} g / \nu D_{C}$.

The location of the thin chemical front is given by $z=h(x, y, t)$, a function of the horizontal coordinates and the time, obeying the eikonal relation ${ }^{3}$

$$
\hat{\mathrm{n}} \cdot \hat{\mathrm{z}} \frac{\partial h}{\partial t}=v_{0}+\mathscr{\mathscr { D }}_{C} \kappa+\left.\hat{\mathbf{n}} \cdot \mathbf{v}\right|_{h} .
$$

Here, $\hat{\mathrm{n}}$ is the normal vector to the front pointing into the unreacted fluid, $v_{0}=(a / \nu) c_{0}$ is the dimensionless flat front speed, $\left.\mathbf{v}\right|_{h}$ is the fluid velocity evaluated at $z=h(x, y, t)$, and $\kappa$ is the curvature of the front measured as positive when the center of curvature is in the unreacted fluid.

The boundary conditions require that the velocity and the normal derivative of the temperature vanish at the sidewalls, ensuring no-slip, insulating sidewalls. The jump conditions at the front ${ }^{3}$ express the conservation of energy, mass and momentum:

$$
\begin{aligned}
& {[\hat{\mathbf{n}} \cdot \mathbf{v}]_{h}=0,} \\
& {[\hat{\mathbf{n}} \times \mathbf{v}]_{h}=0,} \\
& {\left[\epsilon_{i j k} n_{j} n_{l} T_{k l}^{v}\right]_{h}=0,} \\
& {[P]_{h}=-\mathscr{F}_{C} h-\left[n_{i} n_{j} T_{i j}^{v}\right]_{h},} \\
& {[\hat{\mathbf{n}} \cdot \nabla T]_{h}=\frac{\Delta T}{\mathscr{Q}_{T}}\left(\hat{\mathbf{n}} \cdot \hat{z} \frac{\partial h}{\partial t}-\left.\hat{\mathbf{n}} \cdot \mathbf{v}\right|_{h}\right),}
\end{aligned}
$$

and

$$
[T]_{h}=0 .
$$

Here, $[q]_{h}$ denotes the jump in a given function $q=q(\mathbf{x}, t)$ in the following way. If $q(\mathbf{x}, t)=q_{r}(\mathbf{x}, t)$ in the reacted fluid and $q(\mathbf{x}, t)=q_{u}(\mathbf{x}, t)$ in the unreacted fluid, then $\left.[q]_{h} \equiv q_{r}\right|_{h}-\left.q_{u}\right|_{h}$ is the difference between these functions evaluated at the reaction front $z=h$. Furthermore, $T_{i j}^{v}=-\partial v_{i} / \partial x_{j}-\partial v_{j} / \partial x_{i}$ is the (dimensionless) viscous stress tensor, $\epsilon_{i j k}$ is the totally antisymmetric tensor, $n_{i}$ are the Cartesian components of $\hat{\mathrm{n}}$, and $\Delta T=T_{1}-T_{0}$ involves the initial fluid temperature $T_{0}$. Equations (1) determine the convective motion and stability of a thin autocatalytic reaction front.

Steady upward propagation of a convectionless flat front is conveniently described in a frame of reference that is stationary with respect to the front. With the front located at 
$z=h^{(0)}=0$ (and the unreacted fluid at $z>h^{(0)}$ ), Eqs. (1) give $\mathbf{v}^{(0)}=-v_{0} \hat{z}, \quad \hat{\mathbf{n}}^{(0)}=\hat{z}, \quad h^{(0)}=\kappa^{(0)}=0, \quad \partial P^{(0)} / \partial z=\mathscr{S} \mathscr{Q}_{C} \gamma$ $\times\left(T^{(0)}-T_{1}\right)$, as well as temperatures

$$
T_{r}^{(0)}=T_{1}
$$

and

$$
T_{u}^{(0)}=T_{0}+\Delta T e^{-z v_{0} / a_{T}}
$$

in the reacted and unreacted fluids. This solution lacks both fluid motion (in the laboratory frame) and horizontal thermal gradients. Hence, it is applicable to both unbounded geometries and geometries with vertical insulating sidewalls.

To study the stability of the convectionless flat front, we perturb it slightly according to $\mathbf{v}=\mathbf{v}^{(0)}+\mathbf{v}^{(1)}$, with similar forms for the other dependent variables. In evaluating Eqs. (1) for small $h$, it is convenient to employ Taylor expansions,

$$
\left.q\right|_{h}=\left.q\right|_{0}+\left.h \partial_{z} q\right|_{0}+\left.\frac{1}{2} h^{2} \partial_{z}^{2} q\right|_{0}+\cdots
$$

and

$$
[q]_{h}=[q]_{0}+h\left[\partial_{z} q\right]_{0}+\frac{1}{2} h^{2}\left[\partial_{z}^{2} q\right]_{0}+\cdots,
$$

where $\partial_{z}=\partial / \partial z$. Note that even though $q(\mathbf{x}, t)=q_{u}(\mathbf{x}, t)$ only for $z>h(x, y, t)$ and $q(\mathbf{x}, t)=q_{r}(\mathbf{x}, t)$ only for $z<h(x, y, t)$, the functions $q_{r}(\mathbf{x}, t)$ and $q_{u}(\mathbf{x}, t)$ are defined over all space, so that the difference $[q]_{0}=\left.q_{r}\right|_{0}-\left.q_{u}\right|_{0}$ between these functions at $z=0$ is a well-defined quantity. Using these in Eqs. (1) and linearizing in the perturbations yields

$$
\begin{aligned}
& \left(\partial_{t}-v_{0} \partial_{z}-\nabla^{2}\right) \mathbf{v}^{(1)}=\mathscr{P Q S} T^{(1)} \hat{z}-\nabla P^{(1)}, \\
& \left(\partial_{t}-v_{0} \partial_{z}-\mathscr{Z}_{T} \nabla^{2}\right) T^{(1)}+\left(\partial_{z} T^{(0)}\right) w^{(1)}=0 \text {, } \\
& \nabla \cdot v^{(1)}=0 \text {, } \\
& \left(\partial_{t}-\mathscr{Q}_{C} \nabla^{2}\right) h^{(1)}=\left.w^{(1)}\right|_{0}, \\
& {\left[w^{(1)}\right]_{0}=0 \text {, }} \\
& {\left[\hat{\mathrm{z}} \times \mathbf{v}^{(1)}\right]_{0}=0 \text {, }} \\
& {\left[\epsilon_{i 3 k} T_{k 3}^{v(1)}\right]_{0}=0 \text {, }} \\
& {\left[P^{(1)}\right]_{0}=-\mathscr{P} \mathscr{Q}_{C} h^{(1)}+2\left[\partial_{z} w^{(1)}\right]_{0} \text {, }} \\
& {\left[T^{(1)}\right]_{0}=-\frac{\Delta T v_{0}}{\mathscr{D}_{T}} h^{(1)},}
\end{aligned}
$$

and

$$
\left[\partial_{z} T^{(1)}\right]_{0}=\frac{\Delta T}{\mathscr{Q}_{T}}\left(\frac{v_{0}^{2}}{\mathscr{D}_{T}}+\mathscr{\mathscr { L }}_{C} \nabla^{2}\right) h^{(1)}
$$

Here, we have appropriately set $\hat{\mathrm{n}}^{(1)}=-\nabla h^{(1)}$ (a horizontal vector) and $\kappa^{(1)}=\nabla^{2} h^{(1)}$, and have written the vertical velocity as $w^{(1)}=\hat{\mathbf{z}} \cdot \mathbf{v}^{(1)}$.

The unbounded system is the simplest possible geometry in which to study convection in autocatalytic systems. Here we analyze the time dependence of infinitesimal perturbations about a convectionless flat unbounded front. For simplicity, we analyze two-dimensional perturbations $\mathbf{v}^{(1)}(x, z, t)=u^{(1)}(x, z, t) \hat{\mathrm{x}}+w^{(1)}(x, z, t) \hat{z}$ by assigning the exponential dependence $e^{i q x+\sigma t}$ to all perturbed quantities. Substituting these forms into Eqs. (4) yields a system of

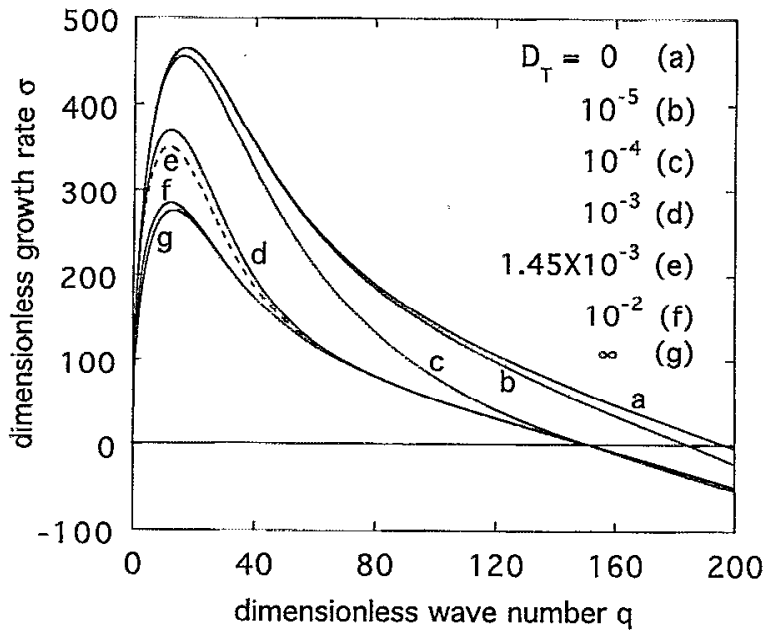

FIG. 1. Dimensionless growth rate $\sigma$ for two-dimensional perturbations of dimensionless wave number $q$ about a flat ascending reaction front in a laterally unbounded geometry for various thermal diffusivities $D_{T}$ measured in units of $\mathrm{cm}^{2} / \mathrm{s}$, with the dashed trace corresponding to the thermal diffusivity of water.

ordinary differential equations that is similar to Eqs. (9) in Ref. 5, except for an important contribution from the discontinuous density. Exponential solutions are readily obtained after the manner of Ref. 5.

Figure 1 shows the resulting growth rate $\sigma$ of infinitesimal perturbations about a flat front as a function of the wave number $q$ for the typical parameters ${ }^{3} D_{C}=2 \times 10^{-5} \mathrm{~cm}^{2} / \mathrm{s}$, $\delta_{1}=0.87 \times 10^{-4}, \Delta T=0.4^{\circ} \mathrm{C}, \quad \nu=9.2 \times 10^{-3} \mathrm{~cm}^{2} / \mathrm{s}$, and $c_{0}=2.95 \times 10^{-3} \mathrm{~cm} / \mathrm{s}$ for various values of the thermal diffusivity $D_{T}$, where the results for $D_{T}=0$ (trace a) and $D_{T}=\infty$ (trace g) are taken from Ref. 3. For the experimental value $D_{T}=1.45 \times 10^{-3} \mathrm{~cm}^{2} / \mathrm{s}$ (trace e), the cutoff wavenumber $q_{c}=152$ where $\sigma=0$ agrees very well with the infinite $D_{T}$ result (trace $\mathrm{g}$ ), indicating an effectively infinite thermal diffusivity. This result can also be seen in Fig. 2. In contrast, the maximum dimensionless growth rate $\sigma_{m}$ and the corresponding wave number $q_{m}$ for this value of $D_{T}$ (trace e) differ significantly from their infinite diffusivity limits. For $q \rightarrow 0$ and this same value of $D_{T}$, the growth rate agrees with the $D_{T}=0$ limit (trace a). Figure 2 emphasizes that $q_{c}$ crosses over from effectively zero to effectively infinite thermal diffusivity at a lower value of $D_{T}$ than $q_{m}$ and $\sigma_{m}$.

To understand the crossover from zero to infinite thermal diffusivity, we need only compare the convective length scale with the thermal length scale. The thermal length scale $d_{T}=D_{T} / c_{0}$ in conventional units [see Eq. (4b)] gives the vertical distance over which the temperature falls from $T_{1}$ to $T_{0}$. The convective length scale $d=\left(\nu / c_{0}\right) 2 \pi / q$ in conventional units is set by the dimensionless horizontal wave number $q$, and gives the vertical extent over which the fluid participates in the convection. In the infinite thermal diffusivity limit $d<<d_{T}$, the fluid participating in the convection is all essentially at the reacted fluid temperature $T_{1}$, and the fluid is unable to take advantage of the thermally induced density changes which are well above the convective region. On the other hand, in the zero thermal diffusivity limit 


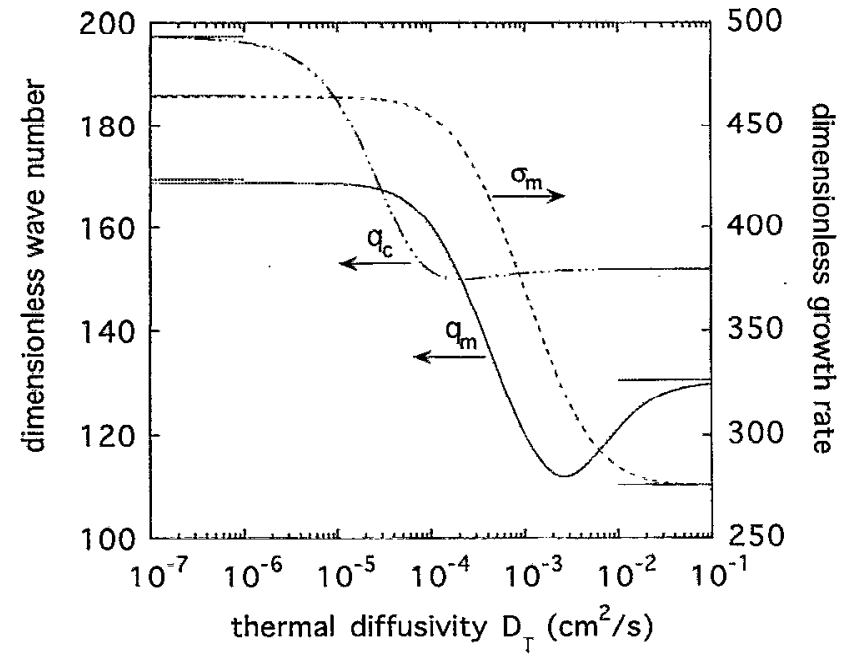

FIG. 2. Maximum dimensionless growth rate $\sigma_{m}$ (dashed trace) and its corresponding dimensionless wave number $q_{m}$ (solid trace), along with the cutoff dimensionless wave number $q_{c}$ (chain-dashed trace) of zero growth rate, all as a function of the thermal diffusivity $D_{T}$. Horizontal line segments give previous asymptotic results for large and small diffusivities. ${ }^{3}$

$d>>d_{T}$, the thermally induced density changes occur over a region small compared with the convective region, which extends well into the unreacted fluid at temperature $T_{0}$. The crossover between zero and infinite diffusivity should therefore occur when $d \approx d_{T}$, that is, when $D_{T}^{\text {cross }} \approx 2 \pi \nu / q$. Since the values $11<q_{m}<17$ of $q_{m}$ are much smaller than the values $150<q_{c}<200$ of $q_{c}$ over the whole range of $D_{r}$ (Fig. 2), the crossover value $D_{T}^{\text {cross }} \approx 4 \times 10^{-3} \mathrm{~cm}^{2} / \mathrm{s}$ for $q_{m}$ must ex-

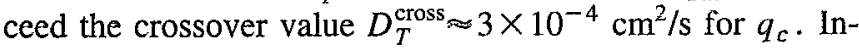
deed, these crossover values are consistent with the crossover behavior in Fig. 2.

Intriguing minima in $q_{c}$ and $q_{m}$ occur in Fig. 2 near the crossover values of $D_{T}$, where the convective and thermal length scales are comparable. At the minimum in $q_{c}$, the flat front is most stable to convective perturbations, and is even more stable than for infinite thermal diffusivity. This is due to thermal stabilization of the perturbed front: Because heat flow is normal to the front surface [Eq. (1i)], the unreacted fluid above the valleys in the front is heated faster than the fluid above the peaks. Thermal expansion consequently renders the fluid above the valleys more buoyant than otherwise, thus tending to raise the valleys and lower the peaks. This stabilizing mechanism is absent at infinite thermal diffusivity where the fluid temperature is effectively uniform throughout. It is also absent at zero thermal diffusivity, where all thermal gradients are confined to the front surface itself. Thermal stabilization is only relevant when the convective and thermal length scales are comparable, where it produces the minima in $q_{c}$ and $q_{m}$.

The two-dimensional motion of a fluid confined between two parallel vertical planes located at $x= \pm a / 2$ and the axisymmetric motion of a fluid confined to the interior of a long vertical cylinder of radius $a$ are studied by generalizing the expansion procedure in Ref. 4 . In each case, a flat ascending front is linearly unstable to convection above a critical length scale $a_{c}$ for the onset of convection (Fig. 3). The limits of

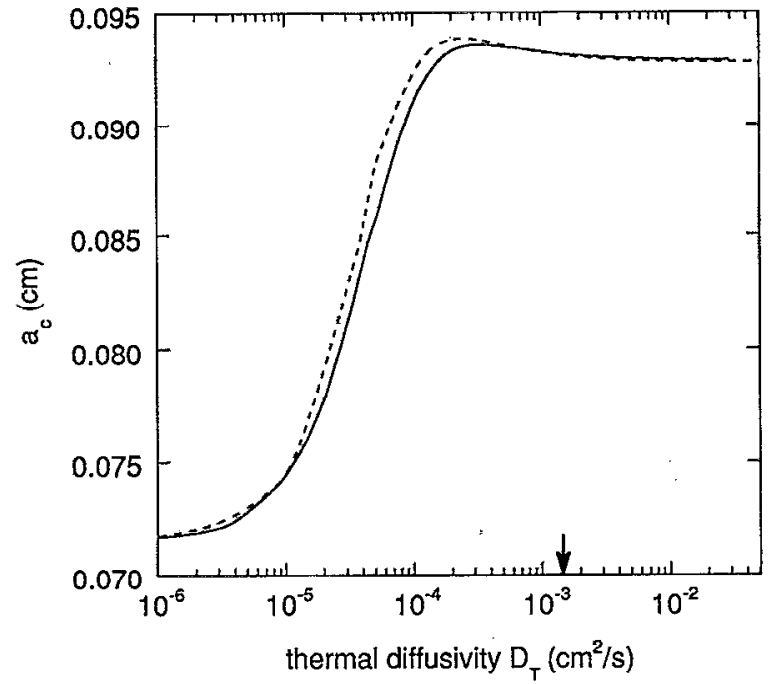

FIG, 3. Critical wall separation $a_{c}$ (solid trace) and tube diameter $a_{c}$ (dashed trace) vs. thermal diffusivity $D_{T}$. The vertical arrow marks the experimental thermal diffusivity $D_{T}=1.45 \times 10^{-3} \mathrm{~cm}^{2} / \mathrm{s}$.

zero and infinite thermal diffusivity agree with our previous calculations. ${ }^{4}$ The maximum in $a_{c}$ implies maximum stability, and corresponds to the minimum in $q_{c}$ discussed earlier. Results for $D_{T}=1.45 \times 10^{-3} \mathrm{~cm}^{2} / \mathrm{s}$ (vertical arrow), can clearly be approximated by the infinite diffusivity limit.

In conclusion, chemical gradients overwhelm thermal gradients in determining the length scale for the onset of convection in autocatalytic reaction fronts. However, at larger convective length scales approaching the thermal length scale, thermal gradients are important. An additional mechanism for stabilizing ascending reaction fronts, relevant only at finite thermal diffusivity, arises from the enhanced (depressed) heating rates above valleys (peaks) in the front, which renders the fluid above the valleys hotter and more buoyant than otherwise. This mechanism may play a crucial role in systems for which chemical and thermal gradients compete rather than cooperate with each other to destabilize the system.

\section{ACKNOWLEDGMENTS}

Discussions with Kenneth Showalter and support from National Science Foundation Grants No. RII-8922106 and No. OSR-9255224 and the National Research Center for Coal and Energy are gratefully acknowledged.

${ }^{\mathrm{I}} \mathrm{T}$. McManus, "Chemical waves in the iodate-arsenous acid reaction," Ph.D. thesis, West Virginia University, 1989, Chap. 3.

${ }^{2}$ J. A. Pojman, I. R. Epstein, T. J. McManus, and K. Showalter, "Convective effects on chemical waves. 2. Simple convection in the iodatearsenous acid system," J. Phys. Chem. 95, 1299 (1991).

${ }^{3}$ B. F. Edwards, J. W. Wilder, and K. Showalter, "Onset of convection for autocatalytic reaction fronts: Laterally unbounded systems," Phys. Rev. A 43, 749 (1991).

${ }^{4}$ D. A. Vasquez, B. F. Edwards, and J. W. Wilder, "Onset of convection for autocatalytic reaction fronts: Laterally bounded systems," Phys. Rev. A 43, 6694 (1991).

${ }^{5}$ J. W. Wilder, B. F. Edwards, and D. A. Vasquez, "Finite thermal diffusivity at onset of convection in autocatalytic systems: Continuous fluid density," Phys. Rev A 45, 2320 (1992). 